\title{
Atividades práticas no ensino de Odontologia Legal nos cursos de graduação em Odontologia
}

\author{
Julia Gabriela Dietrichkeit Pereira*; Karinna Fróes Lima**; Fernanda Braz Romano**; Paula \\ Barreto Costa*; Victor Jacometti*; Ricardo Henrique Alves da Silva*** \\ * Universidade de São Paulo, Faculdade de Medicina de \\ Ribeirão Preto, Programa de Pós-Graduação em \\ Patologia. Aluno de Mestrado (Odontologia Legal) \\ ** Universidade de São Paulo, Faculdade de Odontologia \\ de Ribeirão Preto, Curso de Especialização em \\ Odontologia Legal. Especialista (Odontologia Legal). \\ *** Ribeirão Preto. Professor Doutor (Odontologia Legal)
}

Recebido em 13/01/2017. Aprovado em 06/04/2017.

\begin{abstract}
RESUMO
A Odontologia Legal é, muitas vezes, considerada um componente curricular teórico, cabendo ao docente encontrar recursos para transformá-lo em uma atividade prática, facilitando o aprendizado dos estudantes. $\mathrm{O}$ objetivo deste artigo foi apresentar algumas das atividades práticas no campo da Odontologia Legal realizados na Faculdade de Odontologia de Ribeirão Preto da Universidade de São Paulo (FORP-USP), bem como discutir seus respaldos didáticos no processo de ensino/aprendizagem, enfatizando a relevância das atividades práticas e em grupo no ambiente acadêmico. As atividades práticas apresentadas neste artigo contemplam: (a) arqueologia forense; (b) identificação odontolegal; (c) traumatologia forense; (d) local de crime e estações didáticas. Conclui-se que a realização de atividades práticas na área de Odontologia Legal é viável e aplicável, permitindo aos alunos vivenciar o contexto forense.
\end{abstract}

Descritores: Odontologia Legal. Educação em Odontologia. Docentes de Odontologia. Estudantes de Odontologia.

\section{INTRODUÇÃO}

As aulas tradicionais devem ser contextualizadas a fim de garantir o aprendizado e, mesmo assim, constituem somente um núcleo de ensino, necessitando de mais do que aparatos tecnológicos para serem efetivas, pois precisam ser coesas de maneira a fornecer o produto final, o aprendizado, de maneira completa ${ }^{1}$. Teoria e prática são essenciais em qualquer processo de aprendizado, sendo a última trivial para a construção de um aspecto diferente ao aluno ${ }^{2}$. Soma-se a isso o benefício de despertar o 
interesse do aluno, utilizando instrumentos importantes como o trabalho em conjunto e estudo de casos práticos.

O ensino é um grande desafio ao professor que busca fazer com que seus estudantes sejam capazes de transformar a realidade que os circunda. Para que o sujeito desenvolva esta habilidade, precisa realizar atividades que contenham planejamento definido e objetivos a serem alcançados ${ }^{3}$. Além disso, dinâmicas em grupo auxiliam o ensino, pois evitam o isolamento do estudante, consequentemente facilitando o processo de aprendizagem ${ }^{3,4}$.

Os trabalhos realizados em grupo permitem a construção do saber de maneira coletiva ${ }^{5}$. Destarte, os resultados destes trabalhos são construídos democraticamente entre os integrantes do grupo, situação em que o estudante aprende a ouvir e respeitar o próximo, ao mesmo tempo em que agrega o conhecimento ${ }^{6}$. Esse aspecto é interessante, visto que o aluno do curso de Odontologia é tipicamente técnico e centrado no individualismo, tendo uma formação voltada à clínica e, muitas vezes, descontextualizada da realidade social ${ }^{7}$.

$\mathrm{Na}$ busca de solução para estes entraves, as Diretrizes Curriculares Nacionais (DCN) para os cursos de Odontologia, publicadas em 2002, sugerem alterações na organização dos cursos, fornecendo aos estudantes novas formas de instrução, principalmente pela vivência ${ }^{8}$.

Nesse sentido, a Odontologia Legal, componente curricular da maioria dos cursos de graduação em Odontologia no Brasil, é vista corriqueiramente como sendo burocrática e morosa, por tratar de assuntos supostamente teóricos e distantes da prática odontológica tradicional. No entanto, a gama de assuntos que tem a oferecer é muito ampla, trazendo conteúdo das interfaces com outras áreas forenses ${ }^{9}$. Na atualidade, é notável o número progressivo de profissionais da Odontologia que ingressam em carreiras oficiais forenses, como Peritos Criminais ou Odontolegistas, bem como nas demais atividades profissionais vinculadas a essa área de formação ${ }^{10-12}$.

O objetivo deste artigo foi apresentar uma breve descrição de algumas atividades práticas passíveis de estruturação no contexto do ensino universitário em Odontologia Legal, baseados em relato de experiência de atividades conduzidas na disciplina de Odontologia Legal da Faculdade de Odontologia de Ribeirão Preto da Universidade de São Paulo (FORP-USP), bem como discutir seus respaldos didáticos no processo de ensino/aprendizagem, enfatizando a relevância das atividades práticas e em grupo no ambiente acadêmico.

\section{RELATO DE EXPERIÊNCIA}

$\mathrm{Na}$ disciplina de Odontologia Legal da FORPUSP, algumas atividades práticas são realizadas com os estudantes como dinâmica de grupo, valorizando o conhecimento experiencial, com o aluno participando diretamente da geração do saber $^{13}$. Os alunos, frente a diversos simulacros de situações reais, devem tomar decisões e elaborar relatórios/laudos sobre cada atividade a seguir apresentada.

\section{Arqueologia Forense e Identificação Odon- tolegal}

Uma das possíveis atuações do cirurgiãodentista inserido em forças policiais, na condição de perito, é a identificação humana por meio dos $\operatorname{arcos}$ dentais $^{14}$. Quando se tratarem de fragmentos esqueletizados, não é cabível realizar a papiloscopia, enquanto que a genética forense é, muitas vezes, um meio oneroso e demorado. Assim, a Odontologia Legal, por meio da comparação de dados odontológicos ante mortem e post mortem, desempenha papel fundamental ${ }^{15}$ como método primário de identificação ${ }^{16}$, sendo autossuficiente para estabelecer o vínculo do 
questionado ao suspeito $^{11,16}$.

$\mathrm{Na}$ atividade prática de graduação, os estudantes são conduzidos a um ambiente campestre da FORP-USP, onde se deparam com "locais de crime" pré-demarcados, que devem ser explorados, utilizando técnicas de arqueologia forense e instrumental específico (pás e pincéis).

Devem, então, documentar todo o processo por meio de fotografias e realizar esboço do local examinado, lançando mão de trenas para realizar mensurações e obter a localização topográfica dos achados. Para a realização dessa atividade, são enterrados um crânio e uma mandíbula sintéticos, que devem ser retornados e seus arcos dentais analisados para composição da documentação post mortem.

Em um segundo período didático, são distribuídos a cada grupo quatro prontuários odontológicos que diferem em formato e em conteúdo, sendo que apenas um destes prontuários é compatível com o encontrado no sítio escavado pelo grupo. Os estudantes devem estabelecer a identificação positiva ao prontuário correto e excluir os demais (figura 1). A atividade busca reiterar a possibilidade de atuação do cirurgião-dentista no meio pericial, concebendo a identificação de desconhecidos por meio de processos comparativos.

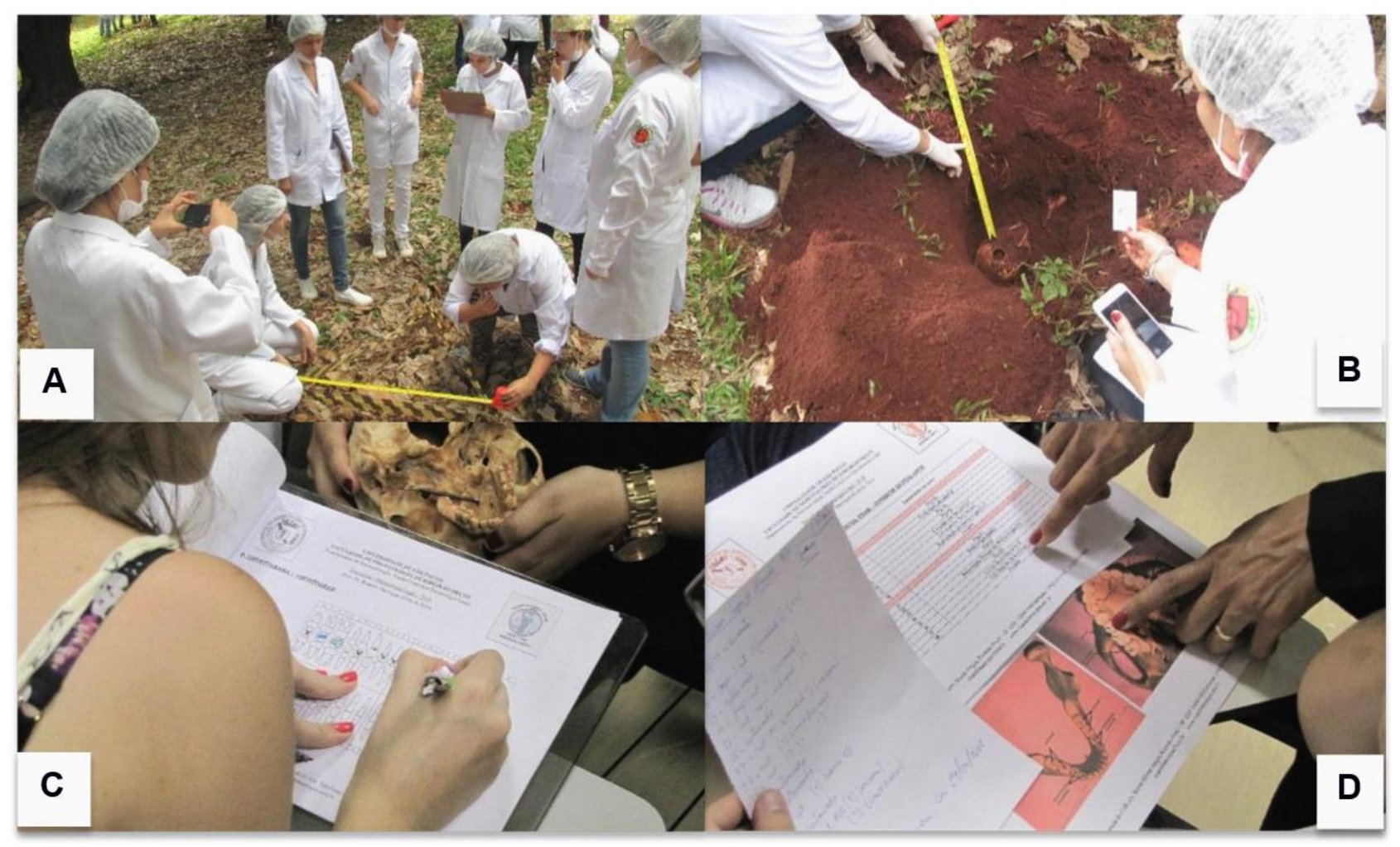

Figura 1 - Atividade prática de Arqueologia Forense e identificação odontolegal: (A) análise do local demarcado; (B) encontro de vestígio; (C) e (D): análise odontolegal post mortem, ante mortem e confronto

\section{Traumatologia Forense}

O ensino da Traumatologia Forense é, na maioria dos casos, teórico e ilustrativo (por meio de fotografias projetadas), distanciando o aluno da vivência prática desse conteúdo. A criação de modelos pode auxiliar a abordagem desse tema, com simulação de lesões em manequins da região de cabeça e pescoço, aproximando os discentes de maneira mais concreta, utilizando-se de recursos visuais como estratégia de ensino. 
A atividade prática realizada consiste na confecção de lesões simuladas em manequins com cabeça e pescoço, para facilitar a visualização, assimilação e fixação de suas nomenclaturas. As lesões fictícias, na referida experiência, são dispostas em seis manequins, onde estão ilustradas 27 diferentes lesões, numeradas de 1 a 27, compatíveis com lesões de energia de ordem mecânica (figura 2). Nessa atividade é possível constatar um maior interesse e motivação dos alunos, além de melhor entendimento e assimilação das nomenclaturas e características das diversas lesões relacionadas a traumatologia forense.
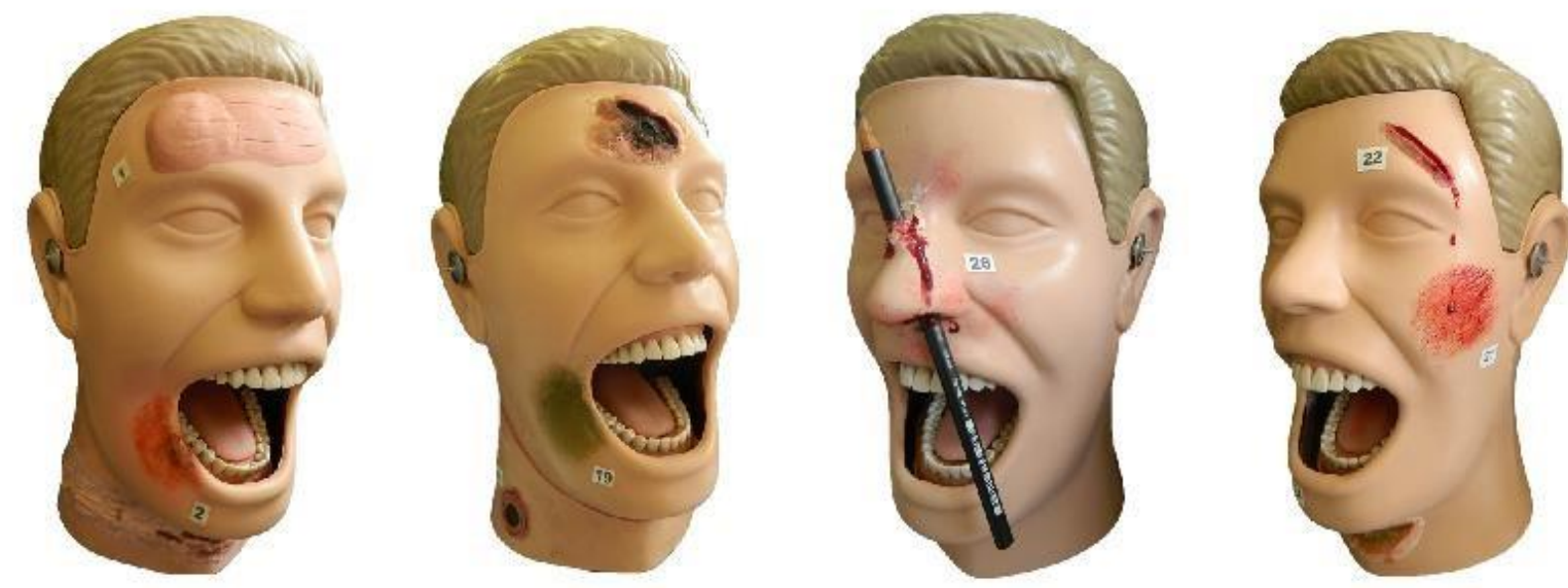

Figura 2 - Manequins modificados com lesões de ordem físico-mecânicas simuladas através de materiais sintéticos (maquiagens, massa de modelar)

\section{Manequins Odontológicos e a Identificação Odontolegal}

O uso dos manequins odontológicos é uma prática já estabelecida nos cursos de Odontologia, porém o seu emprego para atividades que possam auxiliar no desenvolvimento de habilidades práticas na Odontologia Legal ainda é pouco explorado. Uma das possíveis dinâmicas que podem ser desenvolvidas com manequins é a identificação odontolegal. Nesta situação, são planejados os mais diversos tratamentos odontológicos para cada manequim, envolvendo extrações, restaurações, próteses e outros procedimentos, os quais são executados nos elementos dentais artificias.

Posteriormente, são criados prontuários odontológicos que irão conter as informações ante mortem do paciente e, para simular um caso real onde há mais de um possível suspeito para a identificação da vítima, são criados três prontuários contendo informações odontológicas similares, porém com uma ou mais discrepâncias inexplicáveis que promovam a exclusão desse suspeito, direcionando a identificação (figura 3).

$A$ atividade se desenvolve em etapas. Num um primeiro período, os estudantes devem fazer um exame minucioso do manequim para obter as informações post mortem. Em seguida, é feito o confronto das informações obtidas com as informações ante mortem contidas nos prontuários, para, dessa forma, obter uma possível identificação. 

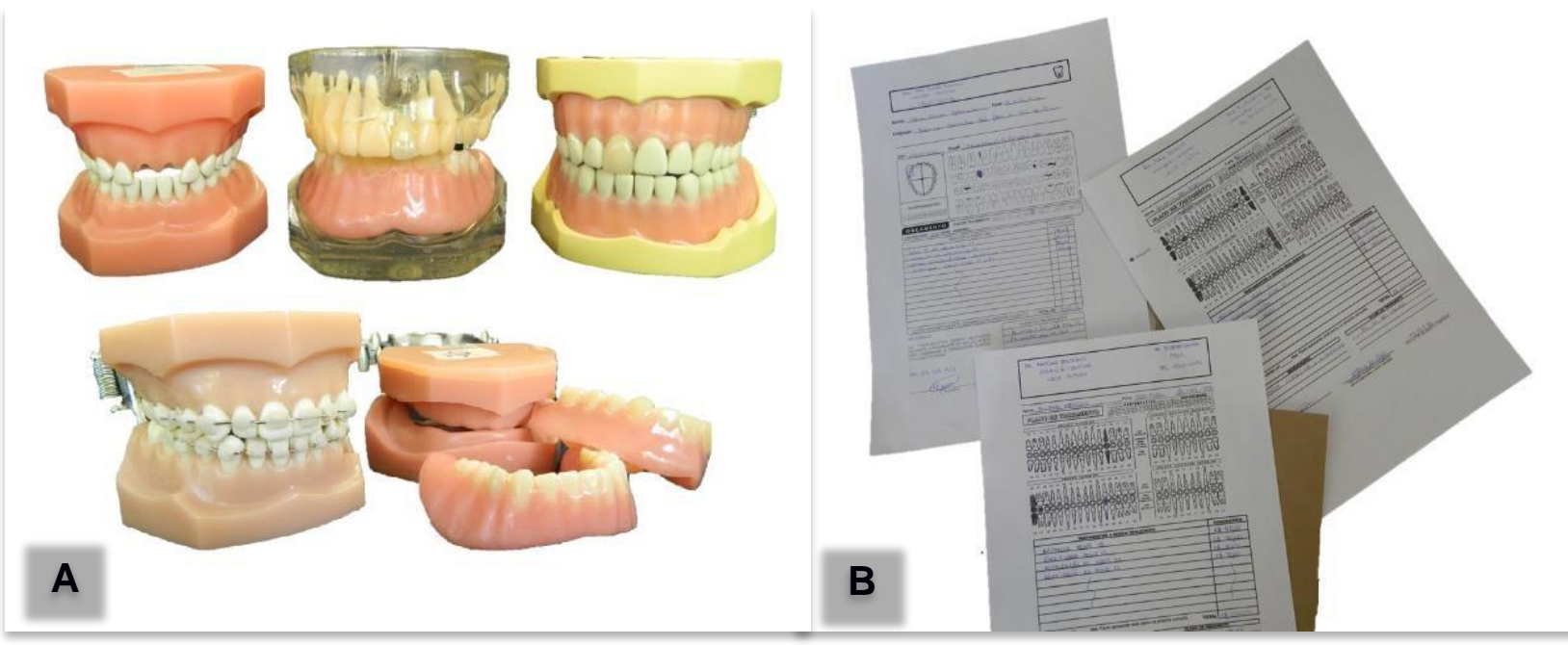

Figura 3 - (A): Manequins odontológicos com diversos tratamentos realizados (restaurações, próteses, extrações, etc.); (B): simulacros de prontuários odontológicos

\section{Local de crime e estações didáticas}

Em uma cena de crime, inúmeros são os vestígios que podem ser localizados. Porém, sem a correta observação do local e um olhar crítico, muitos vestígios podem ser deixados de lado, dificultando a análise acerca da materialidade, autoria e dinâmica do crime cometido ${ }^{17-19}$. Com isso, o objetivo desta atividade é simular um local de crime para os estudantes, onde os mesmos deverão encontrar todos os vestígios para a posterior elaboração de laudo (que se configura como o relatório da atividade).

A atividade é desenvolvida por meio da criação de uma história fictícia, com desfecho criminoso. Logo após, cria-se um local com a presença de vestígios necessários e algumas informações são oferecidas aos participantes para que consigam discernir quais delas são relevantes e quais são irrelevantes para o caso. Após analisar o local do crime, fotografar e anotar o que consideram importante, os estudantes passam por diferentes estações didáticas, nas quais recebem uma curta explanação sobre determinados tipos de vestígio, a importância de como identificá-lo, como coletá-lo e seu uso no processo de investigação. Complementando a atividade, outras estações com temas importantes na área forense, porém sem relação com o local de crime em si, podem ser inseridas com a finalidade de ampliar o aprendizado (figura 4).

\section{CONSIDERAÇÕES FINAIS}

Elaborar processos educativos no escopo da formação de competências é um objetivo que exige inovações pedagógicas ${ }^{3}$ e no caso das atividades práticas aqui apresentadas, estas foram planejadas com base metodológica nas dinâmicas de grupo. Fato posto, pode-se observar que a dinâmica de grupo é uma situação simulada cujo desenvolvimento visa criar experiências para aqueles envolvidos no aprendizado e iniciar nestes seus próprios processos de investigação e aprendizado $^{20}$.

A Odontologia Legal faz parte do conjunto de especialidades odontológicas que estão listadas na Resolução CFO 63/2005, estando em seus artigos 63 e 64, sua definição e áreas de competência $^{21}$. Com as atividades aqui apresentadas, busca-se estabelecer uma proximidade dos estudantes com algumas destas áreas de competência, tais como identificação humana, perícia em foro criminal, elaboração de 
laudos e traumatologia odontolegal ${ }^{9}$. Dessa de análise, interpretação e de descrição em forma, desenvolvem-se habilidades pertencentes relatos e documentos pertinentes ${ }^{22}$, sobre as quais aos princípios da Criminalística - observacionais, exercícios práticos são eficientes e benéficos ${ }^{23}$.

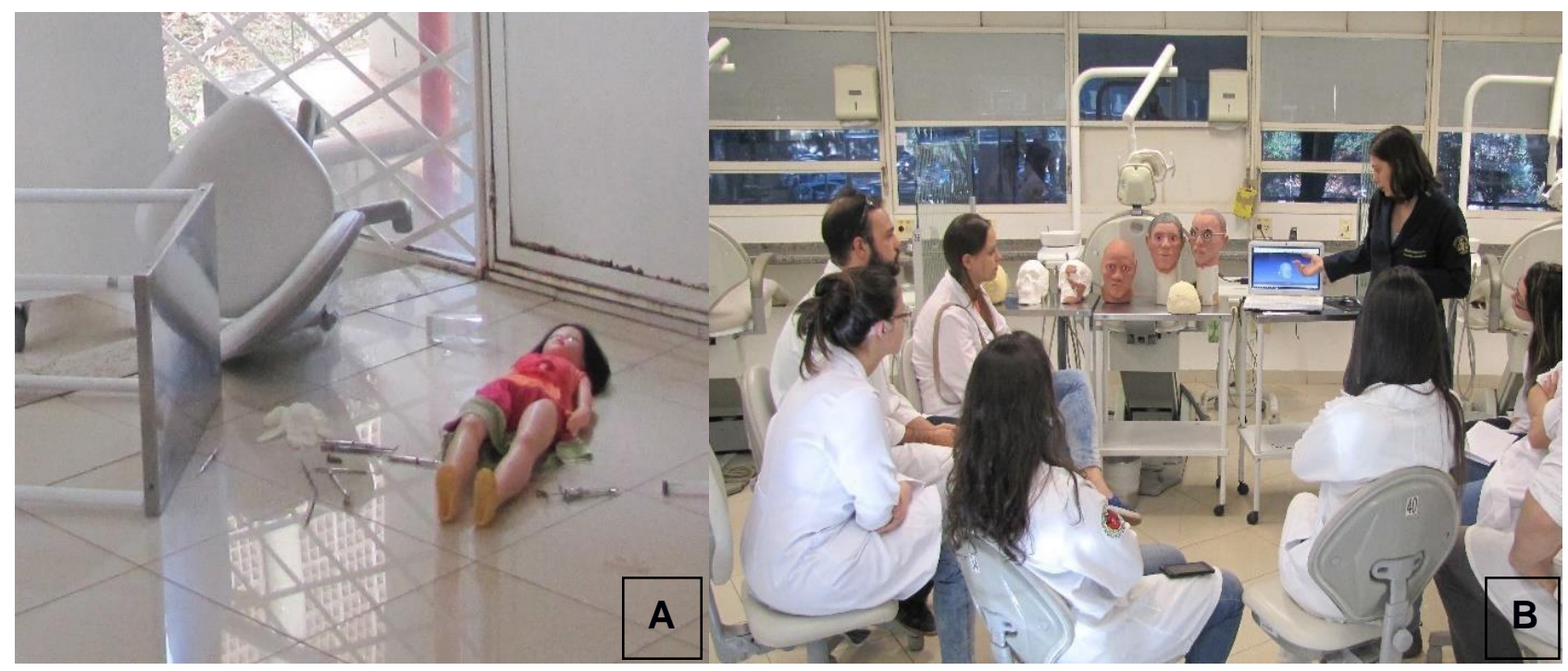

Figura 4 - (A): Local de crime simulado; (B): Estação didática

Estimulando os alunos a resolver situações artificiais similares à realidade, consegue-se fazer com que as atividades práticas sejam memorizadas de maneira mais efetiva ${ }^{24}$, pois aguçam a capacidade criativa, fazendo a aprendizagem se desenrolar coletivamente ${ }^{13} \mathrm{e}$, além disso, instigam a desenvoltura dos participantes melhorando sua produtividade porque visualizam, na prática, suas ações interferindo na realidade e modificando-a em busca do resultado esperado 25 .

Para que estas atividades práticas em grupo atinjam estes objetivos, é necessário um planejamento do que se pretende expor aos estudantes, tendo em vista o conhecimento objetivado a ser absorvido por estes ${ }^{3}$. Nesse processo, alguns contratempos foram encontrados, principalmente o período de duração da aula e o alto número de alunos (em média, oitenta alunos por turma para apenas um docente $)^{26}$.

Apesar disso, a boa infraestrutura oferecida pela FORP-USP e pessoal disponível para apoio técnico, auxiliando na logística das atividades, foram facilidades encontradas. Nesse sentido, pós-graduandos da área de Odontologia Legal da instituição auxiliam no contornar dos obstáculos mencionados, exercendo papel de tutores, permitindo a divisão do grande número de estudantes em grupos menores. Essa colaboração permite atenção individual e assistência ao docente no planejamento e desenvolvimento de seus projetos acadêmicos ${ }^{27}$.

Ainda que o tutor exerça a função de educador neste processo ${ }^{28}$, não suprime a importância do professor, pois a docência exige domínio dos métodos de ensino ${ }^{29}$ e, portanto, demanda saberes complexos, porque engloba a formação humana em toda sua dimensão. Assim, o docente deve apresentar intrínseca conexão entre sabedoria teórica e prática ${ }^{30}$.

Por fim, as atividades práticas com dinâmicas de grupo em ambientes e situações não convencionais não significam apenas a permuta 
do espaço físico ou do material utilizado. Constituem novas relações de conhecimento, sendo o estudante não mais apenas um receptor passivo de informações, tendo uma atitude mais ativa e reflexiva frente ao conhecimento, pois a iniciativa prática estabelece relações entre os fundamentos teóricos e a realidade em $\mathrm{si}^{31}$. Dessa forma, promovem a assimilação do conteúdo de maneira mais motivada e instigante, sendo inclusive preferíveis aos espaços tradicionais de aula, em certos casos $^{32}$.

Conclui-se que a utilização de materiais e metodologias diversos para a realização de atividades práticas na área de Odontologia Legal é viável e aplicável, facilitando a aprendizagem e memorização dos alunos, fazendo com que os mesmos saboreiem a dinâmica da Odontologia no contexto forense da vida real.

\section{ABSTRACT \\ Practical activities in the teaching process of Forensic Odontology in Dental undergraduate courses}

Forensic Odontology in dental graduation courses is usually seen as a theoretical issue, and it is up to the professor to find resources to transform it into a more practical class, facilitating student learning. The objective of this article was to present some of the practical activities carried out in the Forensic Odontology classes at School of Dentistry of Ribeirão Preto - University of São Paulo (FORP-USP), as well as to discuss its role in the processes of teaching and learning, emphasizing the relevance of practical and group activities in the academic environment. Practical activities presented include: (a)

Forensic Archaeology; (b) Dental Identification; (c) Forensic Traumatology; and (d) Crime Scene Investigation and teaching stations. It is concluded that the accomplishment of practical activities in Forensic Odontology is feasible and applicable, bringing a forensic experience to the students.

Descriptors: Forensic Dentistry. Education, Dental. Faculty, Dental. Students, Dental.

\section{REFERÊNCIAS}

1. Ribeiro IL, Medeiros-Júnior A. Graduação em saúde, uma reflexão sobre ensinoaprendizado. Trab Educ Saúde. 2016; 14:33-53.

2. Eder ML, Schwartzman G. Formar o recetar: el aporte de la asesoría pedagógica para transformar las prácticas docentes. Rev Hosp Buenos Aires. 2011;31(3):93-8.

3. Alberti TF, Abegg I, Costa MRJ, Titton M. Dinâmicas de grupo orientadas pelas atividades de estudo: desenvolvimento de habilidades e competências na educação profissional. Rev Bras Estud Pedagog. 2014;95(240);346-62.

4. Gomes PT, Pozzebon PMG. Técnicas de Dinâmica de Grupo. In: Carvalho MCM. Construindo o Saber - Metodologia Científica: Fundamentos e Técnicas. $2^{\mathrm{a}}$ ed. Campinas: Papirus; 1989, pp. 129-35.

5. Schunk DHP. Learning Theories: An Educational Perspective. 6 ed. Boston: Pearson. 2011.

6. Barron B. Achieving Coordination in Collaborative Problem-Solving Groups. J Learning Sci. 2000;9(4):403-36.

7. Emmerich A, Castiel LD. Lagarto e a rosa no asfalto: odontologia dos desejos e das vaidades. In: Botazzo C. De lagartos e rosas. Rio de Janeiro: Fiocruz; 2013.

8. Brasil. Ministério da Educação. Parecer CNE/CES no 1.300/2001. Diário Oficial da União. Brasília; 2001; p. 25. [Acesso em 09 jan. 2017]. Disponível em: http://portal.mec. gov.br/cne/arquivos/pdf/CES1300.pdf.

9. Brites AN, Pithan SA, Nunes MF, Brites IF. Odontologia Legal no ensino superior do Estado do Rio Grande do Sul. Rev ABENO. 2016;16(3):36-45

10. Lima KF, Costa PB, Silva RF, Silva RHA. Regulamentação legal da perícia oficial odontolegal nos estados brasileiros. Rev Bras 
Odontol Legal RBOL. 2017;4(1).

11. Ribas-e-Silva V, Terada ASSD, Silva RHA. A importância do conhecimento especialzado do cirurgião-dentista nas equipes de perícia oficial do brasil. Rev Bras Odontol Legal RBOL. 2015;2(1):68-90.

12. Lino-Júnior HL, Gabriel M, Daruge-Júnior E, Silva RHA. Ensino de Odontologia Legal no Brasil: um convite à reflexão. Rev ABENO. 2015;15(2):38-46 13. André MEDA. Estudo de Caso: Seu Potencial na Educação. Cad Pesqui. 1984;49:51-4

14. Silveira EMSZSF. A importância do odontolegista dentro do Instituto Médico Legal. Rev Bras Med Trab. 2013;11(1):34-9.

15. Magalhães LV, Pacheco KTS, Carvalho KS. O potencial da odontologia legal para a identificação humana das ossadas do Departamento Médico Legal de Vitória/ES. Rev Bras Odontol Legal RBOL. 2015:2(2):5-19.

16. INTERPOL. New INTERPOL DVI Guide 2014. [Acesso em 09 jan. 2017]. Disponível em: http://www.interpol.int/INTERPOL expertise/Forensics/DVI-Pages/DVIguide.

17. Dias-Filho CR, Antedomenico E. A perícia criminal e a interdisciplinaridade no ensino de ciências naturais. Química nova na escola. 2010;32(2):67-72.

18. Monteiro IVP. Vestígios hemáticos no local de crime: Sua importância Médico-Legal. [dissertação]. Porto: Universidade do Porto; 2010.

19. Galvão FGSG. Preservação da cadeia de custódia em vestígios biológicos para fins forenses: Caracterização da situação actual e proposta de critérios de recolha e envio de vestígios biológicos [dissertação]. Lisboa: Universidade de Lisboa; 2006.

20. Silva, JAP. O uso de dinâmicas de grupo em sala de aula: um instrumento de aprendizagem experiencial esquecido ou ainda incompreendido? Saber Científico. 2008;1(2):82-9.

21. Brasil. Conselho Federal de Odontologia. Resolução CFO - 63/2005. Consolidação das normas para procedimentos nos Conselhos de Odontologia. [Acesso em 09 jan. 2017]. Disponível em: http://cfo.org.br/wpc ontent/uploads/2009/10/consolidacao.pdf.

22. Tocchetto D, Stumvoll VP. Criminalística. $6^{\mathrm{a}}$ ed. Campinas: Millenium Editora, 2014.

23. Nóbrega JBM, Costa RC, Lima LNC, Rabello PM, Valença AMG, Santiago BM. Percepção dos graduandos de Odontologia quanto à atividade prática de estimativa de idade realizada em uma disciplina de Odontologia Legal. Rev ABENO. 2016;16(2):83-92.

24. Beaini TL, Zanin AA, Miranda GE, Flores M, Curi JP, Melani RFH. Dinâmica de Perícia em Local de Crime na Disciplina de Odontologia Forense da FO-USP. Rev Grad USP. 2016;1(1):77-81.

25. Sociedade Brasileira de Dinâmicas de Grupo (SBDG). Dinâmica de grupo: conhecendo a história da dinâmica dos grupos no Brasil. Blumenau. SBDG, 2006. [Acesso em 09 jan. 2017]. Disponível em: http://www.sbdg.org. br/abremodulo.aspx? codmodulo=10\&codpa gina $=181$.

26. Universidade de São Paulo. Faculdade de Odontologia de Ribeirão Preto. Projeto Político Pedagógico. 2016. [Acesso em 03 abr. 2017]. Disponível em: http://143.107. 153.201/images/stories/grad/ppp2017.pdf.

27. Cabrero BG, Ceballos SP, Vigil MHG, Niebla JC, Garduño CM, Soto YM et al. Las competencias del tutor universitario: una aproximación a su definición desde la perspectiva teórica y de la experiencia de sus actores. Perfiles Educativos. 2016;38 (151):104-22.

28. Gonzalez, M. Fundamentos da tutoria em 
educação a distância. São Paulo: Avercamp, 32. Masetto MT. Docência universitária: 2005. p. 21. repensando a aula. São Paulo; 2000. [Acesso

29. Pimenta SG, Anastasiou LGD. Docência no ensino superior. São Paulo: Cortez; 2010. em 08 nov. 2016]. Disponível em: http:// www.escoladavida.eng.br/anotacaopu/form

30. Grillo MO. Professor e a docência: o encontro com o aluno. In: Enricone D. Ser professor. Porto Alegre: EDIPUCRS; 2002. pp. 55-66

31. Veiga IP, Resende LMG, Fonseca M. Aula acao\%20de\%20professores/repensando_a_a ula.html. universitária e inovação. In: Veiga IP, Castanho MEL. Pedagogia Universitária: a aula em foco. Campinas, SP: Papirus; 2000. pp. 161-91.

Correspondência para:

Prof. Dr. Ricardo Henrique Alves da Silva email: ricardohenrique@usp.br

USP - Faculdade de Odontologia de Ribeirão Preto Avenida do Café, s/n, Bairro Monte Alegre 14040-904 - Ribeirão Preto/SP 